\title{
Los relieves de la urbanización social de la ciudad: Valparaíso, 1820-1880
}

\author{
Leonardo Cortés Estay ${ }^{1}$
}

Recibido: 10 de agosto de 2014 - Aceptado: 10 de octubre de 2014

\begin{abstract}
Resumen
Este artículo se enfoca en las causas de la localización residencial de la urbanización informal, particularmente en el espacio de las quebradas y sus laderas, en Valparaíso entre los años 1820 y 1880 . El texto busca reconocer el rol estructural de la urbanización informal, producida principalmente por los grupos provenientes de la ruralidad chilena, en la conformación de la ciudad de Valparaíso. Por otro lado, se pretende relevar la relación entre relieve físico del suelo y su valor comercial, entendiéndolo como uno de los principales factores que determinaron, y determinan actualmente, la configuración del suelo urbano en esta ciudad.
\end{abstract}

Palabras clave:Urbanismo, Relieve, Valparaíso, Localización residencial.

\section{Aspects of social development in the city: Valparaiso, 1820-1880}

\begin{abstract}
This article focuses on the causes of informal urbanization settlements, particularly in ravines and hillsides in the case of Valparaiso between 1820 and 1880 . The text is aimed to recognizing the structural role of informal urbanization in shaping the city of Valparaiso mainly by groups from rural Chile. On the other hand, it is aimed to relieving the relationship between physical contour of the land and its commercial value, understanding it as one of the main factors that have determined -and currently determines- the urban land setting in this city.
\end{abstract}

Keywords: Urban development, relief, Valparaiso, residential location.

Chileno. Arquitecto. Magíster en Desarrollo Urbano, Pontificia Universidad Católica de Chile. Académico de la Facultad de Arquitectura, Urbanismo y Paisaje, Universidad Central, Chile. Este trabajo es parte de una investigación mayor, titulada El relieve de la pobreza urbana: conformación de la barriada en Valparaíso, 1820-1880. Tesis para optar al grado académico de Magíster en Desarrollo Urbano, Instituto de Estudios Urbanos y Territoriales, Pontificia Universidad Católica de Chile, 2013. E-mail: Icortese@uc.cl 


\title{
Os relevos da urbanização social da cidade: Valparaiso, 1820-1880
}

\begin{abstract}
Resumo
Este artigo enfoca-se nas causas da localização residencial da urbanização informal, particularmente no espaço dos desfiladeiros e seus riachos, em Valparaíso entre os anos 1820 e 1880.0 texto procura reconhecer o papel estrutural da urbanização informal, produzida principalmente pelos grupos provenientes de vida rural chilena, na conformação da cidade de Valparaíso. Por outro lado, pretende-se destacar a relação entre relevo físico do terreno e seu valor comercial, entendendo-o como um dos principais fatores que determinaram, e determinam atualmente, a configuração do terreno urbano nesta cidade.
\end{abstract}

Palavras-chave: Urbanismo, relevo, Valparaiso, Localização residencial.

Durante los últimos dos años, la ciudad de Valparaíso ha sufrido una serie de desastres que dan cuenta de su vulnerabilidad frente a peligros de diversa índole. Entre otros, se puede señalar el incendio en Rodelillo y Placeres, el derrame y desabastecimiento de agua en los cerros debido a la explosión de la matriz ubicada en el Cerro Ramaditas y, por supuesto, el incendio en la parte alta de la ciudad que afectó principalmente a un gran grupo de habitantes localizados en urbanizaciones informales en las partes menos accesibles de diez cerros. Tampoco podemos olvidar los recurrentes aluviones que se llevan a su paso vidas, viviendas y naturaleza.

Sin embargo, traer a presencia la susceptibilidad frente al riesgo de la ciudad no es el objetivo de este artículo, tampoco el indicar que todos estos desastres tienen por común denominador a sus principales afectados: aquellos grupos humanos de mayor vulnerabilidad. El objetivo principal de este texto es preguntarse respecto a las causas que han permitido que aquellos habitantes de urbanizaciones informales, carentes de servicios y estándares de seguridad mínimos, se localicen en los sectores asociados a mayor riesgo, es decir, las quebradas.

En este sentido, el artículo es retrospectivo, enmarcándose entre la década de 1820, inicio de la apertura a mercados internacionales y crecimiento poblacional significativo de Valparaíso, hasta la década de 1880, momento en que se inician reivindicaciones habitacionales del tipo obrero que caracterizarán una nueva forma de producción urbana en las grandes ciudades de Chile. Enfocándose principalmente en las causas de la localización residencial mediante urbanización informal de población principalmente proveniente de la ruralidad, reconociendo su rol estructural en la conformación de la ciudad de Valparaíso, así como también, la relación entre relieve y valor comercial del suelo, como una de las causas determinantes de la configuración del suelo urbano en esta ciudad. 


\section{De puerto a ciudad-puerto: La base productiva y la población urbana}

Fue durante el periodo 1800-1830 que la nación inicia contactos estrechos con los mercados extranjeros, principalmente europeos. Producto de este encuentro se construyó el perfil de la economía nacional, donde cualquier intento de potenciar una industria interna y las fuerzas productivas del país fue doblegado por una abundante incorporación de mercancías extranjeras, las cuales llegaron al territorio nacional en grandes cantidades, buscando conquistar un mercado nuevo para el cual ya poseían excedentes propios de una fuerza productiva industrializada. La competencia por estos productos de menor coste $y$, a su vez, con precios rebajados por la competencia entre los mercaderes extranjeros, hizo imposible un florecimiento de fuerzas productivas nacionales hasta bien avanzado el siglo XIX. Esta interacción tuvo como resultado la llegada de mercaderes extranjeros, quienes acelerarían el desarrollo de los puertos como espacios de comercio.

La nueva relación productiva basada en exportaciones agrícolas y mineras aumentó la presión sobre los terrenos que parecían no haber sido intensamente explotados durante los años de la colonia. Presión sobre las tierras y recursos materiales, así como sobre los grupos humanos considerados como parte, citando a Marx, del ejército de reserva disperso por el territorio. Forma de vida asociada al "vagabundeo" condicionada y reprimida para transformarla en mano de obra desregularizada (Salazar, 2003). Las medidas de control de población, como el requerimiento de salvoconductos para desplazamiento interurbano, buscaban la creación de una clase trabajadora sobre la que descansó la transformación económica del país. Dicha presión motivó, en buena medida, un recurrente traslado a las ciudades que en el imaginario popular parecían ser las proveedoras de un mejor futuro.

Como sector social, este grupo humano es portador de la cultura rural, incorporándola en los ámbitos de lo urbano, adaptándola a los intersticios de la ciudad. Son la nueva masa humana que constituye los índices de explosión demográfica que caracterizan el periodo a partir de 1820 y, como tales, son portadores de una cultura que se manifestará en la construcción de un nuevo 'otro' urbano, despreciado por la clase dominante, cuyos nombres rememoran la ruralidad y el mestizaje: la Chimba, el Guangualí, el Rancho, la Chingana y la Ramada como expresiones materiales de esta nueva ocupación. Sin embargo, durante el periodo estudiado, entre 1820 y 1880, mientras el desarrollo urbano se acelera y la población de las ciudades aumenta de forma notoria, el grupo humano que la constituyó masivamente no generó una cohesión como la que posteriormente identificaría al obrero como sujeto social, y de mercado, capaz de articular luchas de reivindicación que también se plasmarán en la expresión material de lo urbano. 


\section{Puerto de Valparaíso: Crecimiento y ciudad emergente}

Las ciudades del país sufren un inédito aumento de su población a partir de las primeras décadas del siglo XIX, tendencia que se acentuaría durante el desarrollo del siglo y de la cual Valparaíso no sería la excepción. Durante esta época su población aumentó, hasta llegar a ser una de las principales ciudades del país. De alguna manera, se podría deducir que se asiste, durante este periodo, al nacimiento de la ciudad-puerto, en conjunto con todas las problemáticas que una ciudad posee, y en el marco de un crecimiento acelerado, donde la insuficiencia de su infraestructura le daría el dramatismo con que es recordada ésta y otras ciudades por cronistas de la época.

La población urbana de Valparaíso, para el año 1730 no alcanza los tres mil habitantes y cien años más tarde, la población aumentaría al doble, contando en 1828 con unos seis mil habitantes. Sin embargo, para el año 1856 la cifra sería de cincuenta y dos mil, consiguiendo en treinta años un aumento de alrededor de ocho y media veces su población. Este crecimiento seguirá en aumento, ya que veinte años más tarde, para el año 1876, la población casi doblaría la cifra anterior, llegando a 97.049 habitantes y en los siguientes treinta años, para 1907, esta llegaría a los ciento ochenta mil (Ugarte, 1910).

Si bien este aspecto ha sido relatado por historiadores como Ugarte (1910) y Vicuña Mackenna (1936), es de interés caracterizar este aumento poblacional en relación a la morfología y relieve de la ciudad, el modo en que se fueron ocupando sus espacios y cómo estos factores influyeron de forma particular en Valparaíso. La singularidad de esta ciudad radica en que su relieve determinará una serie de aspectos como son: asignación de valor del suelo; uso del suelo de acuerdo a posibilidad de urbanizar; capacidad de acoger grupos de menores recursos; posibilidades de integración de estos grupos vinculándolos favorablemente con el centro urbano, entre otros. Son estas características las que hacen que la descripción del fenómeno de poblamiento de la ciudad durante el siglo XIX adquiera valor desde el punto de vista urbano.

\section{Desarrollo urbano: Los bordes de la ciudad}

Una realidad transversal e ineludible es la morfológica y topográfica, que configurará las relaciones de funcionamiento entre áreas de residencia y centro urbano, además de los valores de suelo, dando cabida a formas de distribución de la población por grupo socioeconómico. La principal característica de la morfología porteña es la presencia de tres bordes que delimitan el espacio urbano. La primera es el borde mar; la segunda es el borde móvil que presentan las distintas alturas de ocupación de los cerros; 
y un tercer borde, múltiple, son las distintas quebradas que subdividen el espacio de los cerros.

La falta de espacio plano frente al mar, donde se establecerían los primeros asentamientos y el centro urbano de la ciudad se hace pronto evidente. Limitando este espacio, se encuentran los cerros y sus quebradas. Esta geografía es la que caracterizará el desarrollo urbano, y es la que le dará su particularidad a la ciudad. De igual modo, la falta de espacio plano incide en el desigual aumento de valor del suelo del plan, siendo ocupada por actividades relacionadas al comercio, y consecutivamente densificando las partes bajas de los cerros para usos residenciales.

Estas condiciones impuestas por la geografía representan muchas veces desventajas en cuanto a costos de infraestructura y valor de suelos. A la vez que representan, oportunidades para quienes vieron en los espacios de quebradas la posibilidad de permanencia, aunque sea en malas condiciones de habitabilidad, configurando verdaderos "bordes interiores".

Estos bordes internos que caracterizan a Valparaíso, diferencian en cuanto a la relación que la ciudad misma presenta con la población de bajos recursos que llegó desde el campo. De acuerdo a De Ramón, los bordes de Santiago se configuraron como fronteras de intercambio entre una sociedad urbana y otra rural, la que se fue desplazando a medida que la población urbana aumentó, sin relación entre campo y ciudad, en el cual se realizaban actividades particulares que se fueron consolidando en el tiempo. Esta concepción de frontera no es igual en Valparaíso, cuyo límite impreciso, entre urbe y ruralidad, permite el emplazamiento de ranchos y cultivos de escala doméstica. Los viejos habitantes rurales que se instalaron en las quebradas fueron perdiendo contacto directo con la ruralidad, permaneciendo más cerca de la ciudad, pero en una configuración que les permitió sobrevivir, mediante la crianza de animales para consumo familiar. Próximos al centro, pudieron diversificar sus actividades y ofrecer otro tipo de servicios como mano de obra o cargadores de puerto.

Valparaíso posee quebradas cuyas pendientes fueron urbanizadas popularmente, permitiendo actividades semejantes a las realizadas en las barriadas santiaguinas, aunque de forma dispersa, ubicándose en éstas las chinganas, de difícil acceso en la ciudad puerto (Ugarte, 1910). Otro elemento frecuente, es el borde mar, proveyendo de alimentación gratuita, siendo considerado, de acuerdo a Salazar, como la "despensa del pueblo". Sin embargo, el borde fue intervenido reiteradas veces, hasta perder su carácter natural que permitía el uso por parte de población muchas veces sin hogar.

El modo en que se configuran los usos y valores del suelo urbano dependen de la relación con la morfología y relieve. Las variables que se desprenden de esto, son expuestas a continuación. 


\section{Configuración del valor de suelo}

Existe consenso entre los historiadores de Valparaíso (Ugarte, 1910; Vicuña Mackenna, 1884) al señalar que el valor del suelo de la planicie de la ciudad era varias veces mayor que el de los cerros; y en los cerros, el que corresponde a los lomajes, especialmente en su parte baja y próxima al centro urbano, era ampliamente superior al de las quebradas.

Sin embargo, esta visión no es generalizable, pues mediante diversas iniciativas de desarrollo urbano, la presión ejercida por el crecimiento demográfico, la existencia de zonas de riesgo, la colonización del relieve porteño y formación de identidades de los nacientes barrios, los distintos sectores de la ciudad fueron adquiriendo distingos respecto a su valoración (de uso y comercial). Confrontando planimetría y testimonios de diversos autores, podemos reconocer cuáles son los factores determinantes en la valoración del suelo en la ciudad y su influencia en los procesos de urbanización.

A continuación se realiza una sectorización de los suelos de Valparaíso, de acuerdo a 5 variables reconocidas mediante examen de textos, confrontados con su contraparte planimétrica.

Tabla 1

Variables incidentes en el valor del suelo

\begin{tabular}{|c|c|}
\hline Variable & \\
\hline \multirow{3}{*}{ Zonas de Riesgo } & Áreas de inundación (el Almendral). \\
\hline & $\begin{array}{l}\text { Vulnerabilidad del centro urbano (uso de la quebrada de San } \\
\text { Francisco). }\end{array}$ \\
\hline & $\begin{array}{l}\text { Quebradas (Zonas de remoción de suelo, de inundación y de } \\
\text { baja calidad ambiental). }\end{array}$ \\
\hline $\begin{array}{l}\text { Proximidad al centro } \\
\text { urbano }\end{array}$ & $\begin{array}{l}\text { Esta relación determinaría la demanda de suelo para uso } \\
\text { residencial de diversos grupos identitarios (colonias) y } \\
\text { socioeconómicos. }\end{array}$ \\
\hline $\begin{array}{l}\text { Condiciones de } \\
\text { emplazamiento e } \\
\text { higiene }\end{array}$ & $\begin{array}{l}\text { Relacionado a aquellas zonas donde era más fácil asentarse, } \\
\text { cuyos costos no fuesen elevados al momento de acondicionar } \\
\text { el terreno, además de poseer ventajas frente al asoleamiento, } \\
\text { calidad de vistas y de ventilación (recordando lo apremiante del } \\
\text { tema higiénico en la ciudad). }\end{array}$ \\
\hline $\begin{array}{l}\text { Infraestructura } \\
\text { urbana }\end{array}$ & $\begin{array}{l}\text { La cual habilitaría ciertas zonas de la ciudad generando nuevo } \\
\text { suelo urbanizable en el plan, reduciendo las zonas de riesgo, } \\
\text { habilitando zonas urbanizables en el cerro y mejorando las } \\
\text { relaciones distancia / tiempo entre cerro y plan. }\end{array}$ \\
\hline
\end{tabular}




\begin{tabular}{|l|l|}
\hline $\begin{array}{l}\text { Identidades socio- } \\
\text { territoriales }\end{array}$ & $\begin{array}{l}\text { Producto del relieve, las áreas residenciales en los cerros } \\
\text { quedan delimitadas por su propia geografía, permitiendo } \\
\text { generar territorios fácilmente reconocibles de acuerdo a } \\
\text { características específicas de los grupos que los habitan, por } \\
\text { ejemplo, cerros de inmigrantes ingleses, alemanes, etc. }\end{array}$ \\
\hline
\end{tabular}

Fuente: Elaboración propia

Estas distintas variables incidieron en un desarrollo desigual de la ciudad de Valparaíso, reconociéndose espacios que se urbanizaron de forma acelerada (aquellos con menor riesgo, mayor proximidad al centro urbano, con mejores condiciones de emplazamiento, con mayor facilidad de urbanizar y cuyas identidades territoriales favorecieron y permitieron mantener su plusvalía, como el caso del Cerro Alegre y Concepción, lugar residencial de marcada procedencia inglesa y alemana), frente a otros espacios que esperaron décadas para mejorar sus estándares, y que encontrándose a primera vista en condiciones de proximidad al centro urbano, por una situación desfavorable en cuanto a una o un conjunto de las variables citadas anteriormente, su calidad en cuanto a estándares urbanos fue baja.

Entre estos últimos espacios se encuentran las quebradas, las que sin importar su proximidad al centro, se reconocen como espacios de riesgo (remoción en masa e inundaciones), además de albergar población inmigrante rural (la que para la oligarquía representaba un grupo poco deseable en las urbes), y por ser utilizadas muchas veces como vertederos, permitiendo el brote de enfermedades como la difteria.

De acuerdo a las distintas variables que otorgan valor al suelo urbano de Valparaíso, este se encuentra principalmente supeditado por las singulares cualidades de su relieve. De acuerdo a la estructura del puerto, su funcionamiento como espacio comercial, ocupando la extensión del borde mar, implicó un uso de las zonas del plan para todos los establecimientos asociados a esta actividad productiva. Por otro lado, las zonas residenciales fueron ocupando las partes más privilegiadas de los cerros y cercanas al plan, estableciendo formas de comunicación lo más directa posible, en la medida de los recursos de sus habitantes. De este modo, se fueron poblando los sectores mejor emplazados respecto a centralidad, accesibilidad y factibilidad de urbanización.

Sin embargo, los distintos usos que se efectuaron sobre el suelo urbano no se realizaron sobre un espacio en blanco. Toda la extensión urbana y extra urbana se encontraba bajo un régimen de propiedad de la tierra. 


\section{Las transacciones del suelo urbano}

Valparaíso, al igual que el resto de las ciudades del país, posee un temprano mercado de suelos, siendo sometidos a transacciones ya sea de venta, arriendo o bien donaciones a diversas instituciones religiosas, de beneficencia o bien con fines cívicos. Sin embargo, estas transacciones evidencian el valor desigual de los diversos suelos que, en el caso de la ciudad de Valparaíso, se relacionarán directamente con su posibilidad de urbanización. Bajo esta mirada, se hace evidente el bajo valor comercial que tuvo el suelo correspondiente a quebradas, en comparación al del plano de la ciudad, así como el de las lomas de los cerros.

Este hecho adquiere relevancia cuando en el contexto del acelerado desarrollo urbano que tuvo lugar en el Valparaíso decimonónico, da pie a un mercado de suelos cuyas formas de subdivisión, transacción, avalúo y asignación de valor a los predios, determinaron la distribución de los grupos sociales en el espacio urbano.

De acuerdo al Plano Catastral de Valparaíso y el documento de Avalúo Municipal del año 1909, los valores de las propiedades se encuentran altamente estratificados, dependiendo de su centralidad, accesibilidad y consolidación de actividades productivas. De acuerdo a esto, propiedades ubicadas en el centro urbano (Plaza Echaurren) podían valer hasta $\$ 800.000$, mientras que en el sector del Almendral su valor podría alcanzar en casos excepcionales hasta $\$ 140.000$ en propiedades comparables en su tamaño. Finalmente, aquellas propiedades ubicadas en los cerros, cuyo valor oscila entre $\$ 10.000$ a $\$ 50.000$ cuando corresponde a quebradas, no presentan avalúo municipal.

Otro aspecto relevante para reconocer el valor y destino de las distintas propiedades, de acuerdo a las variables indicadas en el punto anterior, son aquellas transacciones documentadas que permitieron la generación, principalmente de infraestructura pública y religiosa. Se señalan algunas:

1. Donación para espacio público correspondiente al actual sector de Alejo Barrios en el cerro Playa Ancha, solicitado por Diego Portales al propietario Fernando Urízar.

2. Compra de suelo para el emplazamiento de equipamiento urbano, como el caso del cementerio protestante de Valparaíso.

3. Compra de espacios religiosos para el emplazamiento de edificación pública, como el caso del actual palacio de Tribunales, situado en la quebrada de San Agustín (calle Tomás Ramos) cuyo previo dueño fue la congregación de los Agustinos. 
4. Donaciones, ventas y arriendos para el emplazamiento de congregaciones religiosas, realizados por los grandes propietarios del suelo de Valparaíso. Y si bien, algunas de estas lograron emplazamientos en cerros, como el monasterio de la Santísima Trinidad en el Cerro Larraín, las quebradas han sido, al parecer, espacios cuyo escaso valor ha permitido que instituciones de carácter religioso se establezcan de forma preferencial. Tales son los casos de la orden de los Agustinos emplazados en la quebrada de San Agustín; los Franciscanos en la quebrada de San Francisco; los Domínicos en la quebrada de San Juan de Dios; o la Union Church ubicada en la quebrada de San Agustín, entre otros.

Sin embargo, son las transacciones no documentadas aquellas que indican de mejor forma, cómo el deprimido valor comercial de las quebradas permitió la localización para la urbanización informal de los sectores más pobres de la sociedad. De este modo podemos encontrar documentos desde los que se puede inferir la presencia, ya sea de apropiaciones de suelo para uso residencial, o bien la modalidad de arriendo a piso, forma de renta o inquilinaje.

¿Por qué la quebrada aparece como un espacio de oportunidad para los sectores más pobres de la ciudad? ¿Por qué su valor comercial es tan bajo? O mejor dicho, ¿qué impide que la quebrada se transforme en un suelo preferente para el mercado de suelos?

\section{La quebrada como límite}

Para comprender el escaso interés de las elites por urbanizar las quebradas como posibles áreas de localización residencial, o productiva, es necesario identificar dos aristas de este problema; estas son: a.- la percepción negativa que se tiene respecto a la quebrada, sus pobladores y sus usos; y b.- la dificultad y altos costos que requiere urbanizar en el contexto de pendientes. Esto se explica a continuación.

\section{a. La percepción negativa en torno a la quebrada}

El fenómeno de crecimiento poblacional y apropiación de espacio desvalorizado para el emplazamiento de población popular, en las mayores ciudades del país, concitó diversas visiones de las autoridades y la elite social, donde, la principal argumentación, posteriormente confirmada por los estudios higienistas, conminaba a sanear estos espacios, especialmente al reconocerse como amenazas al estatus de la ciudad de la clase dominante. Las clásicas referencias de Vicuña Mackenna respecto a la dualidad entre una ciudad culta y una ciudad bárbara, son expresiones que fundamentaron posteriores transformaciones urbanas, cuyo énfasis estaba en delimitar las zonas de la población preexistente y la nueva población urbana. 
Valparaíso no es ajeno a este tipo de observaciones, estando localizada la nueva población urbana (ampliamente desprestigiada) en las zonas de quebradas y las alturas de los cerros. En palabras de Vicuña Mackenna, estas zonas se caracterizan mediante "chozas y tugurios de infeliz apariencia" (1884), o en el caso de Ugarte (1910), como espacios de baja moralidad donde se da rienda suelta a las diversiones populares basadas en el consumo de alcohol, violencia y promiscuidad sexual. Esta estigmatización de determinados territorios se vuelve funcional para resolver problemas como el de los residuos y la vivienda popular, siendo utilizados estos espacios como vertederos y áreas sin mayor control por parte de las autoridades, en evidente abandono. De este modo, se consolida no solo una percepción negativa en cuanto al habitante de la quebrada, sino también, a las actividades que aquí se realizan.

\section{b. La dificultad técnica y económica de urbanizar la quebrada}

Si por un lado existe una percepción negativa respecto al entorno antrópico de la quebrada, por otro, existe una dificultad técnica y económica real para urbanizar estos espacios, revirtiendo sus desventajas para transformarlos en áreas urbanas capaces de sostener vivienda o usos productivos dentro de estándares óptimos de seguridad. Esta es una de las razones por las cuales la quebrada, frente a la urbanización formal de los cerros, se transforma en un límite.

De acuerdo a lo anterior, a pesar de que las quebradas poseen una valiosa proximidad al centro urbano, presentan dificultades como son su peligrosidad (desprendimiento de suelo, inundaciones, etc.), así como ausencia de iluminación óptima, ventilación y una vista panorámica a la ciudad-puerto. Pero, principalmente, una de las mayores dificultades radica en que para generar un suelo estable, adecuado para la tradición constructiva de la elite (acostumbrada a edificar en valles), se requiere una transformación costosa del suelo del cerro, esto quiere decir, generar muros de contención, aterrazamientos, apropiadas vías de conexión y finalmente, los medios para dotar de servicios estas zonas.

De esta forma, la urbanización de la quebrada fue dejada de lado, y relegada a los grupos más populares, quienes, en escasos márgenes de seguridad, habitaron estos espacios. 
Figura 1 Una quebrada de Valparaíso (1841).

Autor: Bartolomé Lauvergne. Esta obra logra reflejar el espacio de la ladera de la quebrada como límite, distancia entre el fondo de la quebrada y el lomaje del cerro.

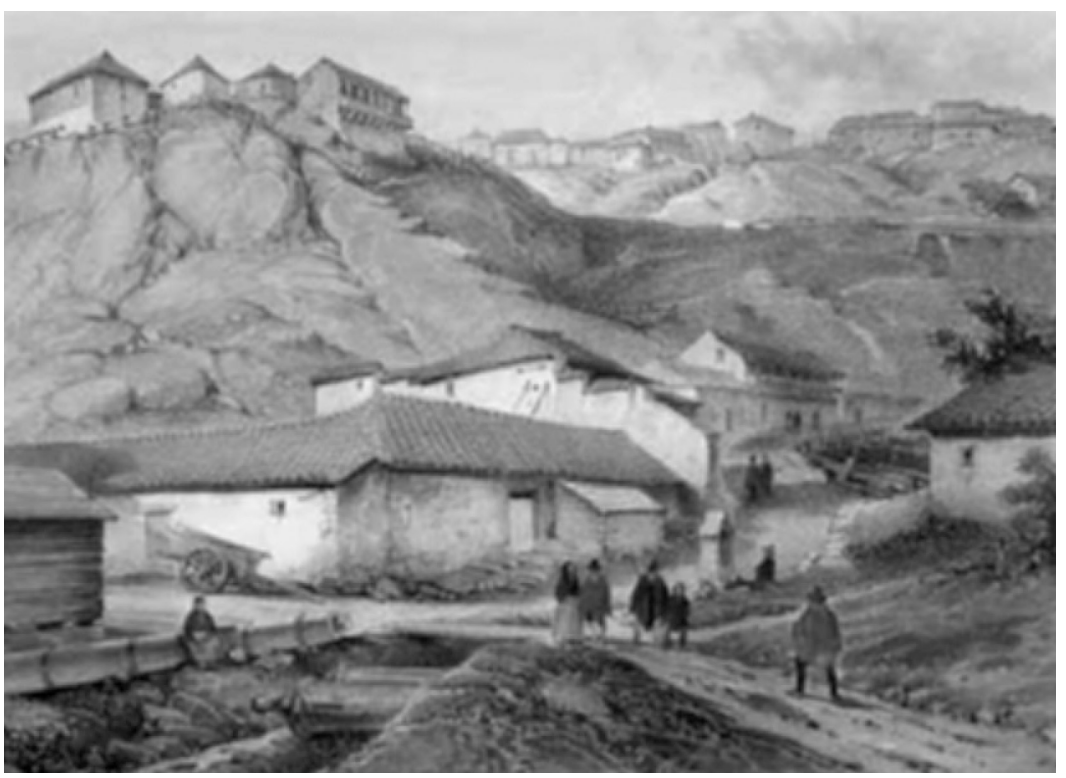

Fuente: El paisaje chileno. Itinerario de una mirada. Museo Histórico Nacional.

Figura 2 Cerro Concepción, 1863. La quebrada como frontera de la urbanización.

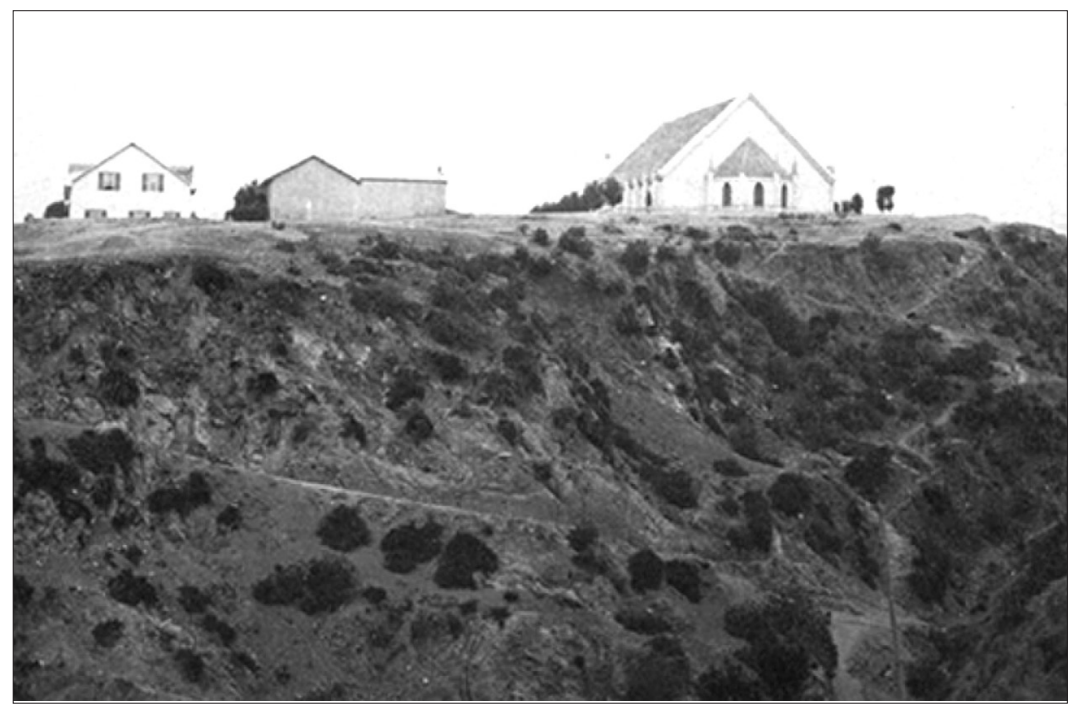

Fuente: Comisión Científica del Pacífico Sur en Chile. 
Figura 3 Cerro Concepción, 1888. Autor: Félix LeBlanc.

Muros de contención que permiten la edificación residencial.

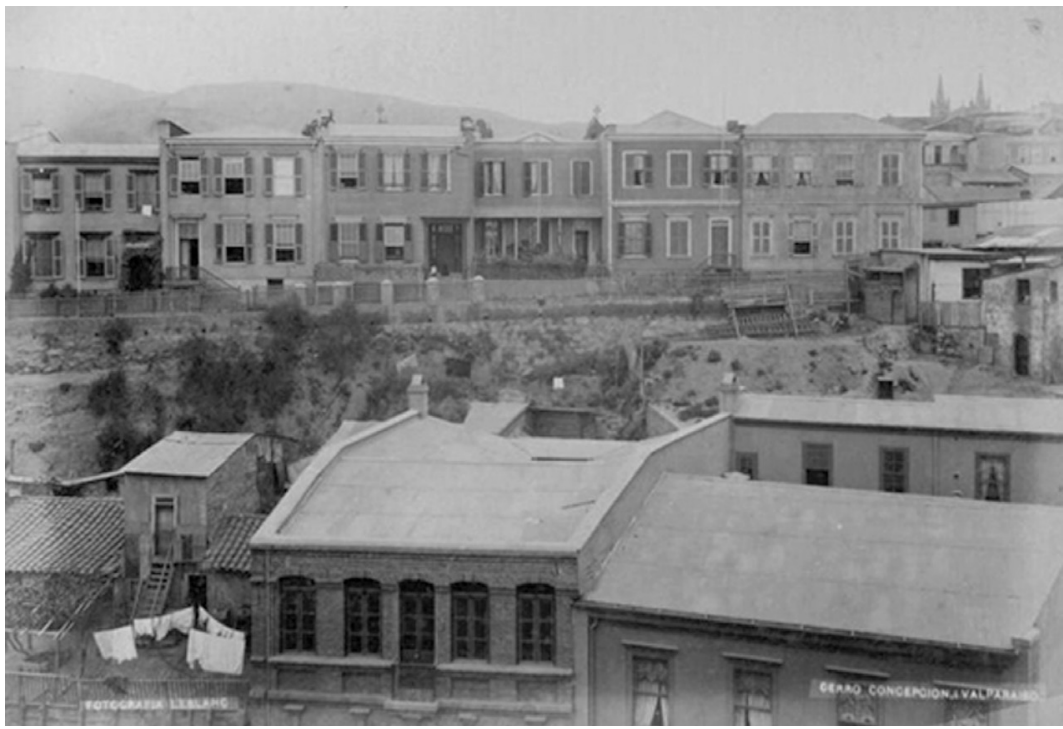

Fuente: Flickr Santiago Nostálgico.

\section{c. La quebrada urbana: Rebautizo-resignificaciones}

Un hecho significativo para la comprensión de la forma de uso de la quebrada en el Valparaíso decimonónico, es el en apariencia anecdótico bautizo que sufren las quebradas una vez que son incorporadas a la ciudad, perdiendo en la planimetría oficial su nombre de quebrada, para ser designada con los nombres de las calles que, una vez reemplacen los cursos de agua originales, ocultándolos bajo las superficies de la nueva urbanidad, serán el vínculo definitivo entre cerro y plano. Respecto a lo anterior, si bien Valparaíso contiene un número permanente de quebradas, su reconocimiento en la planimetría ha sido lento y estas comienzan a ser nombradas, solo una vez que la urbanización las va absorbiendo en el radio urbano.

De acuerdo a Sáez (2001), la planimetría comprendida desde 1854 hasta 1913, va aumentando el número de quebradas, a medida que la urbanización avanza, haciéndolas visibles. El listado inicial de 1854 comprende nueve, las que luego, en el plano de 1876 (elaborado por Santos Tornero), suman veintidós nuevas quebradas, y en el de 1913 (por Santa Ana) agregan cinco quebradas más. Este proceso siguió sumando quebradas durante los años posteriores. Este constante aumento en el reconocimiento de quebradas del entorno urbano, no es indicativo de uso efectivo, sino que se relaciona 
con la visibilidad que la quebrada fue teniendo, a medida que la ciudad formal se extendió por el espacio que actualmente ocupa. Bajo la misma perspectiva, otorgar un nombre a un conjunto de quebradas, dentro de un contexto numéricamente superior, solo puede entenderse como la conciencia que los grupos responsables de estas planimetrías (la intelectualidad y las clases relacionadas a la autoridad) fueron adquiriendo, de territorios que poco a poco se incorporaron a su comprensión del contexto urbano. Del mismo modo se van nombrando quebradas cada vez más altas en el cerro. Sin embargo, se sostiene que el uso de estos espacios, por parte de grupos populares, ya era efectivo desde antes que la autoridad las reconociera e incorporara como referencias urbanas.

De acuerdo a lo anterior, este proceso de "enmascaramiento" que sufre la quebrada, por parte de las autoridades y oligarquía, mediante la suplantación del nombre de la quebrada (significación negativa), por el de la calle (significación urbana positiva) puede interpretarse como una necesidad de expulsar en los nuevos tramos de la ciudad cualquier reminiscencia respecto a lo "exterior", "natural" e incluso "precario" con que habitualmente fueron asociadas las quebradas.

Figura 4 Enmascaramiento de la Quebrada: Quebrada de Jaime - Avenida Francia.

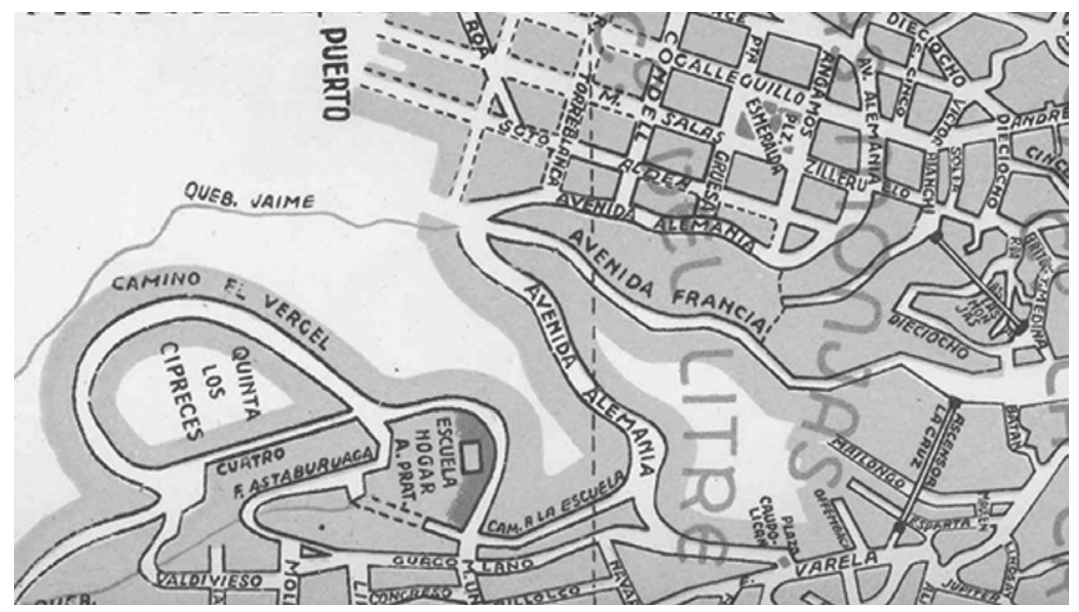

Fuente: Colección personal, Gonzalo Cáceres.

\section{Urbanización formal y urbanización informal del cerro}

De acuerdo al apartado anterior, la incidencia del relieve y los múltiples desafíos técnicos y económicos que presenta al propósito del desarrollo urbano de Valparaíso, sumado al negativo imaginario con que fueron cargados los habitantes de las quebradas, además del uso residual que se le dio, permitieron la creación de dos formas de urbanización. La primera, para 
la elite y las clases más acomodadas, de carácter formal, en localizaciones preferenciales dentro de la difícil geografía del cerro, en las cuales pudiesen reproducir una forma de ciudad que pretende asemejarse a la lógica del modelo hispanoamericano de ciudad de valle, con trazados regulares y en suelos lo más planos posibles. La segunda forma de urbanización es aquella que se localiza en las quebradas y sus laderas, los espacios residuales de la ciudad, alojando a los habitantes más resistidos por las elites, los inmigrantes rurales. Sin resguardos ni estándares de seguridad o servicios, bajo lógicas de autoconstrucción y precariedad material, habitando aquellas zonas que por su dificultad técnica fueron considerados límites.

Algunos ejemplos de la urbanización formal en los cerros los podemos identificar tanto por la planimetría como por la fotografía aérea de algunas de las zonas de más antigua urbanización sobre los cerros. En todos estos casos, se puede reconocer cómo el trazado regular entra en conflicto al enfrentarse a los límites naturales proporcionados por las laderas de los cerros.

Figura 5 Población Bueras, y San Juan del Puerto, 1887.

Fragmento plano Dirección de Obras Públicas de Valparaíso. Son reconocidas como límites las quebradas más abruptas.

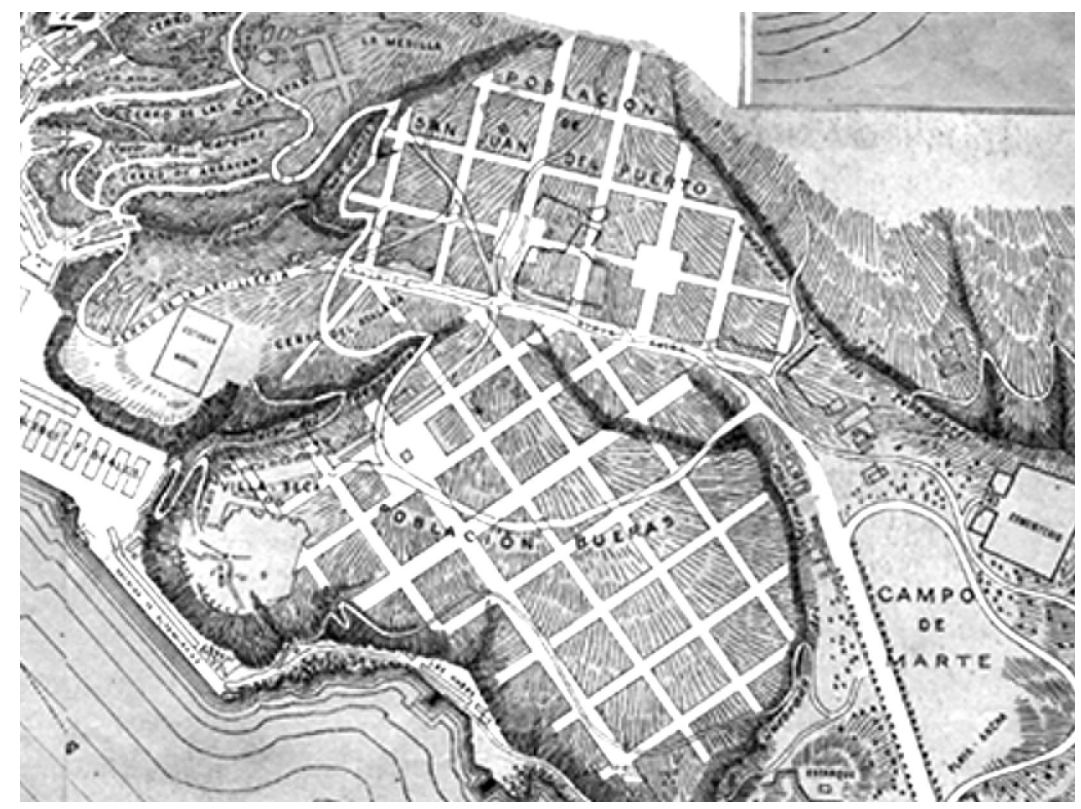

Fuente: Archivo Nacional. 
Figura 6 Izquierda: Trazado regular en Cerro Cordillera.

Derecha: Trazado actual Cerro Barón, persistencia del trazado original.

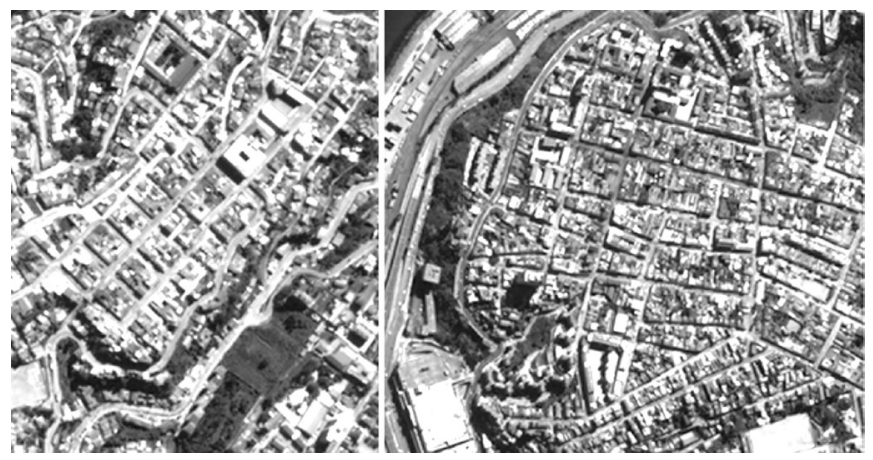

Fuente: Google Maps.

Por otro lado, la urbanización popular de la quebrada posee una lógica distinta en su forma de ocupación de la ladera. Un tema de interés que, sin embargo, no será abordado en este artículo, es el de la correspondencia entre tipología de vivienda en su transformación desde el rancho hasta la vivienda sobre andamios basada principalmente en: (a) presencia de recursos con los que construir; (b) actividades preferenciales transitando entre lo rural a fuentes de ocupación marcadamente urbanas; y (c) densidad residencial sobre la ladera de la quebrada.

Estas diferentes tipologías se corresponden con el nivel de densidad de uso de la ladera, la que comienza a ser habitada de forma más intensiva a medida que se presenta como una oportunidad basada en la proximidad a las fuentes laborales, especialmente las proporcionadas por la actividad portuaria (cargadores, estibadores, etc.). Es en base a esta necesidad de suelo bien emplazado, aunque de difícil uso, que se establecen algunas lógicas generales para la densificación de la ladera, llegando a configurar la idea de mosaico que actualmente reconocemos al mirar los cerros de Valparaíso.

Estos procesos los podemos describir de la siguiente forma:

Tabla 2 Proceso ascendente de ocupación de la ladera

\begin{tabular}{|l|l|}
\hline \multicolumn{2}{|l|}{ Proceso ascendente: } \\
\hline $\begin{array}{l}\text { a.- Linealidad en } \\
\text { torno al cauce de la } \\
\text { quebrada. }\end{array}$ & $\begin{array}{l}\text { Ocupación de los estrechos suelos en el margen del cauce } \\
\text { de agua. Las viviendas, en su mayoría de materialidad sólida } \\
\text { (adobe y teja), siguen la linealidad del curso de agua. }\end{array}$ \\
\hline b.- Ascenso. & $\begin{array}{l}\text { Adosamiento a las viviendas originales, de materialidad y tamaño } \\
\text { variable, así como la orientación respecto al eje de ocupación de } \\
\text { la ladera. }\end{array}$ \\
\hline
\end{tabular}

Fuente: Elaboración propia 
Tabla 3 Proceso descendente de ocupación de la ladera

\section{Proceso descendente}

a.- Las viviendas acomodadas en las zonas privilegiadas del cerro limitan con las laderas, demarcando el comienzo de la quebrada. Por lo general, orientadas a lo largo de este límite.

b.- En una segunda instancia estas viviendas se amplían en su largo, por sobre la pendiente, adosadas a las viviendas originales, sostenidas por estructuras de pilares.

c.- En una tercera instancia comienza el descenso por la ladera, a veces cambiando su orientación y tamaño, descienden soportadas por pilares.

Fuente: Elaboración propia

Tabla 4 Ocupación directa de la ladera

\begin{tabular}{|l|l|}
\hline \multicolumn{2}{|l|}{ Ocupación directa } \\
\hline a.- Aislada & $\begin{array}{l}\text { La vivienda busca un espacio adecuado para emplazarse, } \\
\text { generalmente en forma lineal, siguiendo longitudinalmente la } \\
\text { forma del cerro. Muchas veces es posible observarla agrupada a } \\
\text { otras viviendas, vinculada por caminos que horadan el cerro. }\end{array}$ \\
\hline $\begin{array}{l}\text { b.- En laderas } \\
\text { densamente } \\
\text { edificadas }\end{array}$ & $\begin{array}{l}\text { En un segundo momento, la vivienda más compacta y con menor } \\
\text { espacio para su emplazamiento, se ubica de forma tangencial a } \\
\text { esta, requiriendo una mayor superficie empotrada en estructura } \\
\text { de pilares }\end{array}$ \\
\hline
\end{tabular}

Fuente: Elaboración propia

A continuación se intenta graficar, de forma esquemática, el proceso de urbanización informal de la pendiente, relatado anteriormente:

Figuras 7 - 12

Esquemas respecto a la ocupación de las quebradas.

a. Proceso ascendente

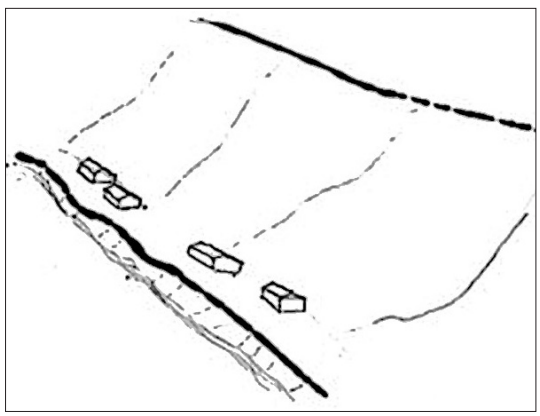




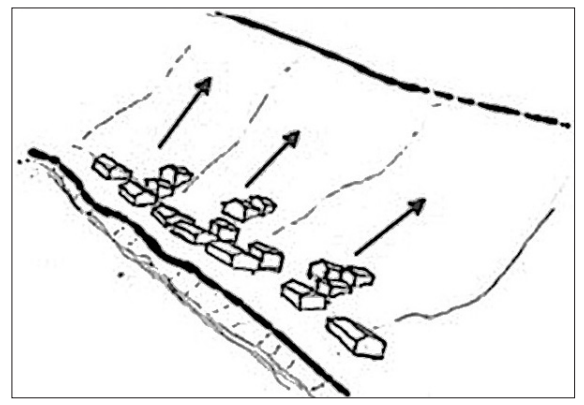

b. Proceso descendente
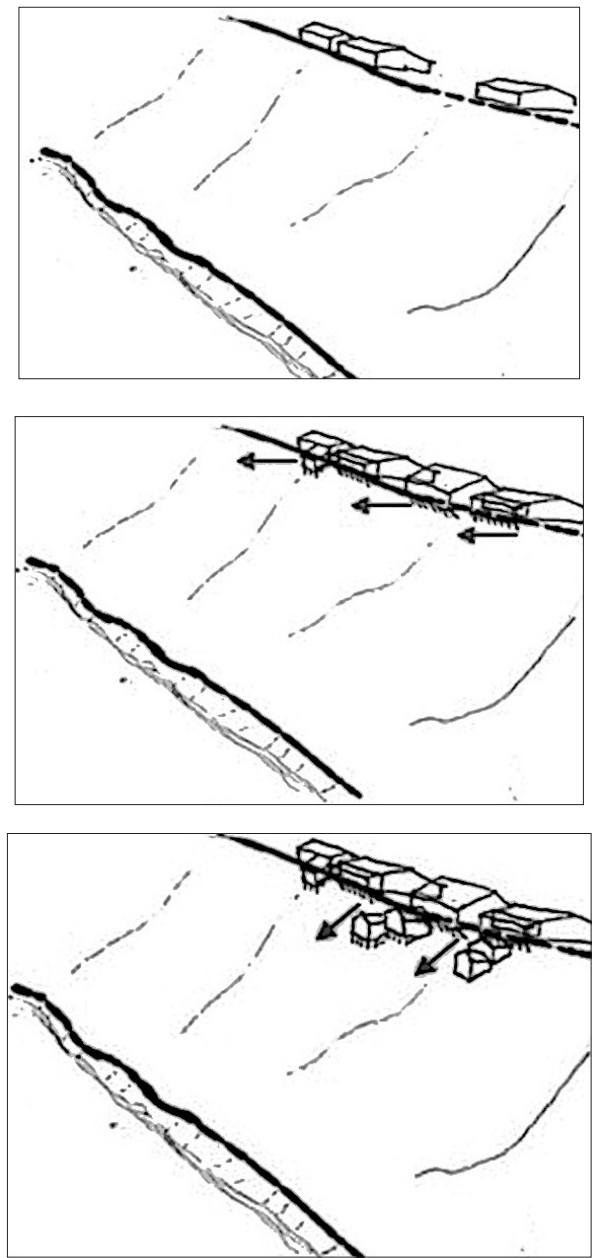
c. Ocupación directa de la ladera

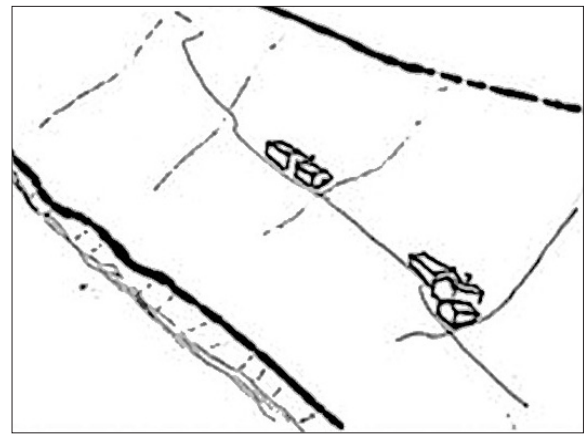

Fuente: Elaboración propia

Este proceso no se dio por separado y demoró un tiempo considerable, configurando una estructura no siempre identificable a una regularidad geométrica.

Figuras $13-14$

Esquemas de poblamiento mediante la totalidad de procesos.

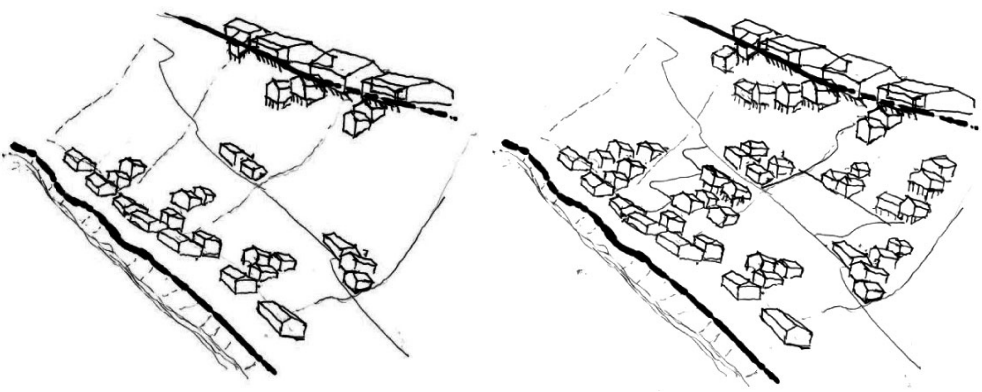

Fuente: Elaboración propia.

\section{Reflexiones finales: Los relieves de la ciudad, espacios de oportunidad para la ciudadanía no reconocida}

A modo de conclusión, es necesario indicar que la ciudad no es un espacio homogéneo, por lo tanto, presenta una serie de particularidades. En el caso de Valparaíso y sin duda otras ciudades de relieves marcados, el espacio de las quebradas ha logrado constituirse como un espacio de oportunidad para aquellos grupos de habitantes con menores recursos económicos. 
Estos espacios, al haber quedado excluidos por largo tiempo del mercado de suelos, han permitido la permanencia de grupos humanos que de otra forma, estarían confinados a los límites externos de la ciudad. Estos habitantes, sin lugar a dudas, han contribuido a su desarrollo, entregando su energía en forma de mano de obra. Por otro lado, se han visto beneficiados por esta proximidad a las fuentes de trabajo, logrando movilidad social, formando actualmente parte del tejido humano "oficial" de la ciudad.

Esta carta de ciudadanía siempre ha sido un elemento de tensión entre poblador de urbanización informal y el Estado, el que siempre ha buscado erradicarlos de la ciudad formal, desconociendo su aporte a la construcción de ésta. Esta tensión se repite bajo distintos discursos o argumentos, ya sean incendios, seguridad ciudadana, higiene, salud pública, etc. Últimamente, el grupo humano que habita la quebrada se ha visto amenazado bajo la forma de la depredación de suelo por parte del mercado inmobiliario, el que ya está en posición de hacer rentable el, hasta ahora, despreciado espacio de la quebrada. Esta acción se ve reforzada por las operaciones de erradicación por parte del Estado a nuevos conjuntos de vivienda social, los que, por supuesto, no ofrecen ninguna ventaja de localización a los habitantes de la quebrada, disminuyendo su posibilidad de movilidad social.

Sin dudas, con estas líneas se plantea una discusión, la cual debe desarrollarse como parte de una contra argumentación que haga más fecunda la toma de decisiones respecto a lo que sucede en la ciudad, especialmente, con ocasión de sus recurrentes desastres.

\section{Referencias bibliográficas}

CARVALLO, D. (1903). "Memoria presentada al Consejo Superior de Higiene por el doctor Daniel Carvallo", en: Consejo de Higiene de Valparaíso. Archivos del Consejo de Higiene de Valparaíso. Valparaíso: El Consejo, 1896-1903.

CAVIERES, E. (1988). "La formación y crecimiento de un mercado moderno: Comercio y Finanzas en Valparaíso", en: Comercio chileno y comerciantes ingleses 1820-1880 (un ciclo de historia económica). Valparaíso: Universidad Católica de Valparaíso, Instituto de Historia.

CLARO, L. (1902). Explicaciones de Derecho Civil Chileno Volumen III. Santiago: Editorial Jurídica de Chile.

DE RAMÓN, A. (1985). "Estudio de una periferia urbana. Santiago de Chile 1850 - 1900", en Historia, N²0, Instituto de Historia, Pontificia Universidad Católica de Chile, p. 199-289. 
DIBAM (2011). El paisaje chileno. Itinerario de una mirada. Santiago: Museo Histórico Nacional, DIBAM.

DIRECCIÓN DE OBRAS PÚBLICAS (1876). Tomo II, Enero 1872 - Diciembre 1875. Imprenta El Mercurio.

GRAHAM, M. (1956). Diario de su residencia en Chile (1822) y de su viaje al Brasil (1823). Santiago: Editorial del Pacífico.

KAPSTEIN, P. (2010). Vulnerabilidad y periferia interior. Madrid: Cuaderno de Investigación Urbanística, Nº71.

MANHEIM, J. B. (1876). Estudio crítico sobre la evacuación general de la ciudad de Valparaíso. Memoria presentada en 1875 al señor intendente Echaurren. Valparaíso: Imprenta del Deber.

MARTLAND, S. (2002). "Cuando el gas pasó de moda: la elite de Valparaíso y la tecnología urbana, 1843-1863", en EURE, Volumen 28, No 83, p. 67-81.

MÉNDEZ, L. (1986). "Plazas y parques de Valparaíso. Transformaciones en el micro paisaje urbano", en Valparaíso 1536-1986; Primera Jornada de Historia Urbana. Valparaíso: Editorial Altazor.

S./A. (1860). Equidad en los impuestos. Valparaíso: Mercurio de Valparaíso.

PORTALES, D. (s/f). "Los problemas políticos de Chile; Documentos oficiales y administrativos", en Ideas y confesiones de Portales (1954). Santiago: Editorial del Pacífico.

RODRÍGUEZ, A. y C. Gajardo (1906). La catástrofe del 16 de agosto de 1906 en la República de Chile. Santiago: Imprenta y Litografía Barcelona.

SÁEZ, L. (2001). Valparaíso: lugares, nombres y personajes, siglos XVI-XXI. Valparaíso: Editorial Puntángeles.

SALAZAR, G. (2000). Labradores, peones y proletarios. Santiago: Editorial LOM.

SALAZAR, G. (2007). Mercaderes, empresarios y capitalistas (Chile, Siglo XIX). Santiago: Editorial Sudamericana.

UGARTE, J. (1910). Recopilación histórica, comercial y social. Valparaíso: Imprenta Minerva.

URBINA, X. (2002). Los conventillos de Valparaíso. 1880 - 1920. Fisonomía y percepción de una vivienda popular urbana. Valparaíso: Ediciones Universitarias de Valparaíso.

VICUÑA MACKENNA, B. (1884). Valparaíso y los ingleses en tres siglos. Santiago: Imprenta Cervantes. 